УДК 629.7.025.6

(C) 2013

Костенко Е. М., кандидат технических наук

Полтавская государственная аграрная академия

\title{
ПЕРЕЧИСЛЕНИЕ ТИПОВЫХ ПЛАНОВ МНОГОФАКТОРНОГО ЭКСПЕРИМЕНТА
}

\section{Рецензент - доктор технических наук, профессор А. Ю. Соколов}

\begin{abstract}
Запропоновано метод перерахування типових оптимальних планів БФЕ, заснований на поданні планів БФЕ у вигляді серійних послідовностей. Розглянуто рішення поетапних задач методу. Досліджено властивості серійних послідовностей планів БФЕ, отримані оичнки їхньої кількості, описана процедура формування множини базових серійних послідовностей $і$ типових варіантів структур планів БФЕ. Наведено приклади каталогів оптимальних планів БФЕ. Застосування запропонованого методу дасть змогу спростити процес вибору мінімальних планів БФЕ для великої кількості факторів.
\end{abstract}

Ключевые слова: фактор, многофакторный эксперимент, перечисление, типовые варианты, структура плана.

Постановка проблемы. Для повышения эффективности исследований, направленных на идентификацию и оптимизацию различных объектов, большое значение имеет разработка стратегии оптимального планирования эксперимента [6]. Построение оптимальных планов многофакторного эксперимента (МФЭ), учитывающих порядок чередования уровней изменения факторов, относится к сложным комбинаторным задачам, и в настоящее время отсутствуют эффективные методы ее решения.

Анализ последних исследований и публикаций по данной проблеме. На стоимость реализации эксперимента существенное влияние оказывает порядок чередования уровней изменения факторов, поскольку различные факторы требуют разных затрат на изменение уровней. При активном эксперименте экспериментатор может менять значения факторов по заданной программе путем изменения порядка выполнения опытов, т.е. производя перестановку строк матрицы планирования эксперимента. В результате этих преобразований формируются новые планы МФЭ, имеющие, в общем случае, различные стоимости изменения уровней факторов в процессе проведения эксперимента. С ростом количества факторов значительно растет количество возможных преобразований планов МФЭ, что затрудняет поиск оптимальных планов. Одним из возможных подходов к решению проблемы является создание справочниковкаталогов с типовыми решениями [7].

Исследованию комбинаторных планов, учитывающих порядок чередования уровней изменения факторов, посвящены работы [1-3], в которых рассмотрено применение комбинаторного анализа при выборе оптимальных планов многофакторного эксперимента [1], эквивалентные преобразования комбинаторных планов многофакторного эксперимента [2]. Методы классификации комбинаторных планов МФЭ исследованы в работе [3]. Известные методы позволяют строить каталоги типовых планов МФЭ, но эффективны только для планов эксперимента с двумя уровнями значений факторов и количеством опытов не более восьми.

Задача оценки количества типовых вариантов оптимальных планов МФЭ и определения их вида относится к классу перечислительных задач. Существующие методы перечисления комбинаторных объектов (теория перечисления Пойа, метод непосредственных подсчетов, асимптотические методы) [6, 7] не учитывают специфику планов МФЭ.

В связи с этим возникает необходимость разработки новых методов перечисления типовых планов МФЭ, позволяющих создавать каталоги типовых решений.

Цель работы: разработка метода перечисления типовых планов МФЭ, учитывающих порядок чередования уровней изменения факторов на основе теории серийных последовательностей.

\section{Результаты исследований.}

1. Представление планов МФЭ в виде серийных последовательностей

При решении задачи перечисления типовых оптимальных планов МФЭ, учитывающих порядок чередования уровней изменения факторов, предлагается представлять планы МФЭ в виде серийных последовательностей. Суть этого представления состоит в следующем. Рассмотрим план МФЭ, приведенный в таблице 1. 


\section{1. План МФЭ}

\begin{tabular}{|c|c|c|c|c|c|c|c|c|c|}
\hline № & $\mathrm{X}_{1}$ & $\mathrm{X}_{2}$ & $\mathrm{X}_{3}$ & $\mathrm{X}_{4}$ & № & $\mathrm{X}_{1}$ & $\mathrm{X}_{2}$ & $\mathrm{X}_{3}$ & $\mathrm{X}_{4}$ \\
\hline 1 & -1 & 1 & -1 & 1 & 9 & -1 & 1 & -1 & -1 \\
\hline 2 & -1 & -1 & 1 & 1 & 10 & 1 & 1 & -1 & -1 \\
\hline 3 & 1 & -1 & 1 & 1 & 11 & 1 & 1 & 1 & -1 \\
\hline 4 & 1 & -1 & 1 & -1 & 12 & -1 & 1 & 1 & -1 \\
\hline 5 & 1 & -1 & -1 & -1 & 13 & -1 & -1 & 1 & -1 \\
\hline 6 & -1 & -1 & -1 & -1 & 14 & -1 & 1 & 1 & 1 \\
\hline 7 & -1 & -1 & -1 & 1 & 15 & 1 & 1 & -1 & 1 \\
\hline 8 & 1 & -1 & -1 & 1 & 16 & 1 & 1 & 1 & 1 \\
\hline
\end{tabular}

Значения факторов в процессе проведения эксперимента можно рассматривать как некоторые последовательности значений (обозначены $\mathrm{P}(\mathrm{X})$ ):

$\mathrm{P}\left(\mathrm{X}_{1}\right)=-1,-1,+1,+1,+1,-1,-1,+1,-1,+1,+1$, $-1,-1,-1,+1,+1$;

$\mathrm{P}\left(\mathrm{X}_{2}\right)=+1,-1,-1,-1,-1,-1,-1,-1,+1,+1,+1$, $+1,-1,+1,+1,+1$;

$\mathrm{P}\left(\mathrm{X}_{3}\right)=-1,+1,+1,+1,-1,-1,-1,-1,-1,-1,+1$, $+1,+1,+1,-1,+1$;

$\mathrm{P}\left(\mathrm{X}_{4}\right)=+1,+1,+1,-1,-1,-1,+1,+1,-1,-1,-1$, $-1,-1,+1,+1,+1$.

Объединим следующие подряд одинаковые значения в группы:

$\mathrm{P}\left(\mathrm{X}_{1}\right)=(-1,-1),(+1,+1,+1),(-1,-1),(+1),(-1)$, $(+1,+1),(-1,-1,-1),(+1,+1)$;

$\mathrm{P}\left(\mathrm{X}_{2}\right)=(+1),(-1,-1,-1,-1,-1,-1,-1),(+1,+1$, $+1,+1),(-1),(+1,+1,+1)$;

$\mathrm{P}\left(\mathrm{X}_{3}\right)=(-1),(+1,+1,+1),(-1,-1,-1,-1,-1,-1)$, $(+1,+1,+1,+1),(-1),(+1)$;

$\mathrm{P}\left(\mathrm{X}_{4}\right)=(+1,+1,+1),(-1,-1,-1),(+1,+1),(-1,-1$, $-1,-1,-1),(+1,+1,+1)$.

Поскольку в пределах групп значения не меняются, то оставим в каждой группе только по одному символу:

$\mathrm{P}\left(\mathrm{X}_{1}\right)=(-1),(+1),(-1),(+1),(-1),(+1),(-1),(+1)$;

$\mathrm{P}\left(\mathrm{X}_{2}\right)=(+1),(-1),(+1),(-1),(+1)$;

$\mathrm{P}\left(\mathrm{X}_{3}\right)=(-1),(+1),(-1),(+1),(-1),(+1)$;

$\mathrm{P}\left(\mathrm{X}_{4}\right)=(+1),(-1),(+1),(-1),(+1)$.

Обозначим переход от значения (-1) к значению $(+1)$ символом $\uparrow$, а переход от значения $(+1)$ к значению (-1) символом $\downarrow$, тогда последовательности можно представить в виде совокупности указанных символов:

$\mathrm{P}\left(\mathrm{X}_{1}\right)=\uparrow \downarrow \uparrow \downarrow \uparrow \downarrow \uparrow$

$\mathrm{P}\left(\mathrm{X}_{2}\right)=\downarrow \uparrow \downarrow \uparrow ;$

$\mathrm{P}\left(\mathrm{X}_{3}\right)=\uparrow \downarrow \uparrow \downarrow \uparrow ;$

$\mathrm{P}\left(\mathrm{X}_{4}\right)=\downarrow \uparrow \downarrow \uparrow$.

Приведенное символьное представление для двух значений уровней показывает только изменения значений в последовательности. Рассмот- ренные последовательности относятся к серийным последовательностям, в которых в серию (группу) входят одинаковые символы.

В результате изменения порядка выполнения опытов меняется вид последовательностей изменения уровней факторов, соответствующих серийных последовательностей и суммарная стоимость изменений. При указанном представлении задача поиска оптимального варианта плана МФЭ сводится к анализу и поиску соответствующих серийных последовательностей.

Анализ известных публикаций показывает, что в настоящее время исследованы свойства отдельных видов символьных последовательностей, которые не учитывают свойства планов МФЭ. В связи с этим были исследованы общие свойства символьных последовательностей.

2. Свойства серийных последовательностей

Пусть имеется алфавит $\Omega=\left\{\omega_{1}, \ldots, \omega_{\mathrm{r}}\right\}$, состоящий из $\mathrm{r}$ символов. Символьной $(\mathrm{r}, \mathrm{n})$ последовательностью называется последовательность $\mathrm{P}=\left\{\mathrm{p}_{1}, \ldots, \mathrm{p}_{\mathrm{n}}\right)$, в которой $\mathrm{p}_{\mathrm{i}} \in \Omega, \mathrm{i}=1, \ldots, \mathrm{n} ; \mathrm{n} \geq \mathrm{r}$ и в последовательности Р представлены все символы из алфавита $\Omega$.

Подпоследовательность $\mathrm{p}_{\mathrm{t}+1} \mathrm{p}_{\mathrm{t}+2 \ldots} \mathrm{p}_{\mathrm{t}+\mathrm{v}}$ называется серией в Р, если

$$
\mathrm{p}_{\mathrm{t}+1}=\mathrm{p}_{\mathrm{t}+2}=\ldots=\mathrm{p}_{\mathrm{t}+\mathrm{v}}
$$

$\mathrm{p}_{\mathrm{t}} \neq \mathrm{p}_{\mathrm{t}+1} ;$ при $\mathrm{t} \geq 1$;

$\mathrm{p}_{\mathrm{t}+\mathrm{v}} \neq \mathrm{p}_{\mathrm{t}+\mathrm{v}+1} ;$ при $\mathrm{t}+\mathrm{v} \leq \mathrm{n}$.

Число символов в серии называется длиной серии, і-ю серию будем описывать в виде $\mathrm{S}_{\mathrm{i}}\left(\mathrm{a}_{\mathrm{i}}, \mathrm{v}_{\mathrm{i}}\right)$, где $\mathrm{a}_{\mathrm{i}}-$ символ, образующий $\mathrm{i}-ю$ серию, $\mathrm{v}_{\mathrm{i}}$ - длина i-й серии. Символьную $(\mathrm{r}, \mathrm{n})$ последовательность $\mathrm{P}$, состоящую из $\mathrm{h}$ серий, будем называть $(\mathrm{r}, \mathrm{n}, \mathrm{h})$ серийной последовательностью и представлять в виде:

$$
\begin{aligned}
& \mathrm{P}=\mathrm{S}_{1}\left(\mathrm{a}_{1}, \mathrm{v}_{1}\right) \mathrm{S}_{2}\left(\mathrm{a}_{2}, \mathrm{v}_{2}\right) \ldots \mathrm{S}_{\mathrm{h}}\left(\mathrm{a}_{\mathrm{h}}, \mathrm{v}_{\mathrm{h}}\right), \\
& \text { где } \mathrm{a}_{\mathrm{i}} \in \Omega \\
& \sum_{\mathrm{i}=1}^{\mathrm{h}} \mathrm{v}_{\mathrm{i}}=\mathrm{n}
\end{aligned}
$$

Множество $\mathrm{A}=\left\{\mathrm{a}_{1}, \mathrm{a}_{2}, \ldots, \mathrm{a}_{\mathrm{h}}\right\}$ называется 
структурой серийной последовательности, а множество $\mathrm{V}=\left\{\mathrm{v}_{1}, \mathrm{v}_{2}, \ldots, \mathrm{v}_{\mathrm{h}}\right\}$ - составом серийной последовательности.

Серия $\mathrm{S}_{\mathrm{j}}\left(\mathrm{a}_{\mathrm{j}}, \mathrm{v}_{\mathrm{j}}\right)$ называется серией i-го вида, если $\mathrm{a}_{\mathrm{j}}=\omega_{\mathrm{i}}$. Количество серий і-го вида $\gamma_{\mathrm{i}}$ и количество символов і-го вида $\lambda_{\mathrm{i}}$ в последовательности Р определяются следующим образом:

$$
\begin{aligned}
& \gamma_{\mathrm{i}}=\sum_{\mathrm{j}=1}^{\mathrm{h}} \beta_{\mathrm{j}}, \lambda_{\mathrm{i}}=\sum_{\mathrm{j}=1}^{\mathrm{h}} \mathrm{v}_{\mathrm{i}} \beta_{\mathrm{j}}, \\
& \beta_{\mathrm{j}}=\left\{\begin{array}{l}
1, \text { если } \mathrm{a}_{\mathrm{j}}=\omega_{\mathrm{i}}, \\
0, \text { в противном случае }
\end{array}\right\} .
\end{aligned}
$$

Множества $\Gamma=\left\{\gamma_{1}, \gamma_{2}, \ldots, \gamma_{\mathrm{r}}\right\}$ и $\Lambda=\left\{\lambda_{1}, \lambda_{2}, \ldots, \lambda_{\mathrm{r}}\right\}$ обладают следующими свойствами:

$$
\sum_{\mathrm{i}=1}^{\mathrm{r}} \gamma_{\mathrm{i}}=\mathrm{h}, \sum_{\mathrm{i}=1}^{\mathrm{r}} \lambda_{\mathrm{i}}=\mathrm{n} \text {. }
$$

Две серийные последовательности относятся к одному типу, если они имеют одинаковую структуру.

3. Перечисление типовых серийных последовательностей планов МФЭ

Серийные последовательности планов МФЭ имеют особенности, обусловленные свойствами планов МФЭ.
При $\mathrm{r}=2$ алфавит $\Omega$ состоит из двух символов $\Omega=\left\{\omega_{1}, \omega_{2}\right\}$, которые чередуются в последовательности. Применительно к планам МФЭ, в которых факторы принимают два значения, алфавит имеет вид: $\Omega=\{-1,+1\}$.

При $\mathrm{k}$ факторах и количестве уровней $\mathrm{r}=2$ количество опытов $\mathrm{n}=2^{\mathrm{k}}$. Обозначим количество серий, образованных символами "-1", через $\gamma^{-}$, а образованных символами "+1" - через $\gamma^{+}$; количество символов "-1" в последовательности обозначим через $\lambda^{-}$, а символов "+1" - через $\lambda^{+}$. Множества $\Gamma=\left\{\gamma^{-}, \gamma^{+}\right\}$и $\Lambda=\left\{\lambda^{-}, \lambda^{+}\right\}$обладают следующими свойствами:

1) $\lambda^{-}=\lambda^{+}=2^{\mathrm{k}-1}$

2) $\gamma^{-}+\gamma^{+}=h$.

Минимальное количество серий $\mathrm{h}_{\min }=2$, a максимальное количество серий $\mathrm{h}_{\max }=\mathrm{n} / 2$.

Значения $\gamma^{-}$и $\gamma^{+}$зависят от значения $\mathrm{h}$ и вида типовой структуры последовательности (А). В таблице 2 приведен пример типовых структур $(2,8)$ последовательностей.

В таблице 3 приведены значения $\gamma^{-}$и $\gamma^{+}$в зависимости от значения $\mathrm{h}$ и вида типовой структуры последовательности (А).

\section{2. Типовые структуры $(2,8)$ последовательностей}

\begin{tabular}{|c|c|c|c|c|c|c|c|c|}
\hline № & $\mathrm{a}_{1}$ & $\mathrm{a}_{2}$ & $\mathrm{a}_{3}$ & $\mathrm{a}_{4}$ & $\mathrm{a}_{5}$ & $\mathrm{a}_{6}$ & $\mathrm{a}_{7}$ & $\mathrm{a}_{8}$ \\
\hline 1 & -1 & +1 & & & & & & \\
\hline 2 & +1 & -1 & & & & & & \\
\hline 3 & -1 & +1 & -1 & & & & & \\
\hline 4 & +1 & -1 & +1 & & & & & \\
\hline 5 & -1 & +1 & -1 & +1 & & & & \\
\hline 6 & +1 & -1 & +1 & -1 & & & & \\
\hline 7 & -1 & +1 & -1 & +1 & -1 & & & \\
\hline 8 & +1 & -1 & +1 & -1 & +1 & & & \\
\hline 9 & -1 & +1 & -1 & +1 & -1 & +1 & & \\
\hline 10 & +1 & -1 & +1 & -1 & +1 & -1 & & \\
\hline 11 & -1 & +1 & -1 & +1 & -1 & +1 & -1 & \\
\hline 12 & +1 & -1 & +1 & -1 & +1 & -1 & +1 & \\
\hline 13 & -1 & +1 & -1 & +1 & -1 & +1 & -1 & +1 \\
\hline 14 & +1 & -1 & +1 & -1 & +1 & -1 & +1 & -1 \\
\hline
\end{tabular}

3. Значения $\gamma^{-}$и $\gamma^{+}$в зависимости от значения $h$ и вида типовой структуры последовательности

\begin{tabular}{|c|c|c|c|}
\hline $\mathrm{h}$ & $\mathrm{A}$ & $\gamma^{-}$ & $\gamma^{+}$ \\
\hline $2 \times \tau$, & $-1,+1, \ldots,-1,+1$ & $\mathrm{~h} / 2$ & $\mathrm{~h} / 2$ \\
\cline { 2 - 4 }$\tau=1, \ldots, 2^{\mathrm{k}-1}$ & $+1,-1, \ldots,+1,-1$ & $\mathrm{~h} / 2$ & $\mathrm{~h} / 2$ \\
\hline $\mathrm{h}=2 \times \tau+1$, & $-1,+1, \ldots,+1,-1$ & {$[\mathrm{~h} / 2]+1$} & {$[\mathrm{~h} / 2]$} \\
\cline { 2 - 4 }$\tau=1, \ldots, 2^{\mathrm{k}-1}-1$ & $+1,-1, \ldots,-1,+1$ & {$[\mathrm{~h} / 2]$} & {$[\mathrm{h} / 2]+1$} \\
\hline
\end{tabular}


Количество $(2, \mathrm{n})$ последовательностей $\mathrm{W}_{\mathrm{i}}$, с типовой структурой $\mathrm{A}_{\mathrm{i}}$, множествами $\Gamma_{\mathrm{i}}=\left\{\gamma_{\mathrm{i}}{ }^{-}\right.$, $\left.\gamma_{\mathrm{i}}^{+}\right\}$и $\Lambda_{\mathrm{i}}=\left\{\lambda_{\mathrm{i}}^{-}, \lambda_{\mathrm{i}}^{+}\right\}$определяется следующим образом:

$$
\begin{aligned}
& \mathrm{W}_{\mathrm{i}}=\delta\left(2^{\mathrm{k}-1}, \gamma_{\mathrm{i}}^{-}\right) \times \delta\left(2^{\mathrm{k}-1}, \gamma_{\mathrm{i}}^{+}\right), \\
& \text {где } \delta(t, d)=\frac{(t-1) !}{(d-1) !(t-d) !} .
\end{aligned}
$$

Количество типовых $(2, \mathrm{n})$ последовательностей $\mathrm{G}(2, \mathrm{n})=2^{\mathrm{k}+1}-2$, следовательно, общее количество $(2, \mathrm{n})$ последовательностей $\mathrm{W}(2, \mathrm{n})$ :

$$
\mathrm{W}(2, \mathrm{n})=\sum_{\mathrm{i}=1}^{2^{\mathrm{k}+1}-2} \delta\left(2^{\mathrm{k}-1}, \gamma_{\mathrm{i}}^{-}\right) \times \delta\left(2^{\mathrm{k}-1}, \gamma_{\mathrm{i}}^{+}\right)
$$

Для построения множества типовых структур $(2, n)$ последовательностей необходимо сформировать последовательности следующего вида:
a) $\mathrm{P}=\vartheta\left(\tau \mathrm{P}^{-+}\right), \tau=1, \ldots, 2^{\mathrm{k}-1}$;
б) $\mathrm{P}=\vartheta\left(\tau \mathrm{P}^{+-}\right), \tau=1, \ldots, 2^{\mathrm{k}-1}$;
в) $\mathrm{P}=\vartheta\left(\tau \mathrm{P}^{-+}, \mathrm{P}^{-}\right), \tau=1, \ldots, 2^{\mathrm{k}-1}-1$;
г) $\mathrm{P}=\vartheta\left(\tau \mathrm{P}^{+-}, \mathrm{P}^{-+}\right), \tau=1, \ldots, 2^{\mathrm{k}-1}-1$,

где $९\left(\mathrm{P}^{1}, \mathrm{P}^{2}\right)$ - операция склеивания последовательностей, в результате которой формируется новая последовательность, образованная из последовательно соединенных последовательностей $\mathrm{P}^{1}$ и $\mathrm{P}^{2}$;

$$
\mathrm{P}^{-+}=(-1,+1) ; \mathrm{P}^{+-}=(+1,-1) ; \mathrm{P}^{+}=(+1) ; \mathrm{P}^{-}=(-1)
$$

$\tau \mathrm{P}$ означает, что последовательность образована из $\tau$ последовательно соединенных последовательностей $\mathrm{P}$.

В общем случае, процедура построения каталога типовых планов МФЭ состоит в генерации множества преобразований, оценки характеристик планов МФЭ, полученных в результате преобразований, и формирования множества различных представителей для типовых планов МФЭ, имеющих наименьшее количество изменений

\begin{tabular}{|c|c|c|c|c|c|c|c|c|c|c|c|c|c|c|c|}
\hline \multirow{3}{*}{$\begin{array}{c}\text { № } \\
1 \\
\end{array}$} & \multirow[b]{3}{*}{2} & \multicolumn{5}{|c|}{ A } & \multirow{2}{*}{$\begin{array}{c}\text { № по- } \\
\text { следова- } \\
\text { тельности }\end{array}$} & \multirow{2}{*}{\multicolumn{8}{|c|}{ Вид последовательности }} \\
\hline & & \multirow{2}{*}{$\frac{a_{1}}{-1}$} & \multirow{2}{*}{$\begin{array}{l}\mathrm{a}_{2} \\
+1\end{array}$} & \multirow[t]{2}{*}{$a_{3}$} & \multirow[t]{2}{*}{$\mathrm{a}_{4}$} & \multirow[t]{2}{*}{$\mathrm{a}_{5}$} & & & & & & & & & \\
\hline & & & & & & & 1 & -1 & -1 & -1 & -1 & 1 & 1 & 1 & 1 \\
\hline 2 & 2 & +1 & -1 & & & & 1 & 1 & 1 & 1 & 1 & -1 & -1 & -1 & -1 \\
\hline \multirow{3}{*}{3} & \multirow{3}{*}{3} & \multirow{3}{*}{-1} & \multirow{3}{*}{+1} & \multirow{3}{*}{-1} & & & 1 & -1 & 1 & 1 & 1 & 1 & -1 & -1 & -1 \\
\hline & & & & & & & 2 & -1 & -1 & 1 & 1 & 1 & 1 & -1 & -1 \\
\hline & & & & & & & 3 & -1 & -1 & -1 & 1 & 1 & 1 & 1 & -1 \\
\hline \multirow{3}{*}{4} & \multirow{3}{*}{3} & \multirow{3}{*}{+1} & \multirow{3}{*}{-1} & \multirow{3}{*}{+1} & & & 1 & 1 & -1 & -1 & -1 & -1 & 1 & 1 & 1 \\
\hline & & & & & & & 2 & 1 & 1 & 1 & -1 & -1 & -1 & -1 & 1 \\
\hline & & & & & & & 3 & 1 & 1 & -1 & -1 & -1 & -1 & 1 & 1 \\
\hline \multirow{3}{*}{5} & \multirow{3}{*}{4} & \multirow{3}{*}{-1} & \multirow{3}{*}{+1} & \multirow{3}{*}{-1} & \multirow{3}{*}{+1} & & 1 & -1 & -1 & 1 & 1 & -1 & -1 & 1 & 1 \\
\hline & & & & & & & 2 & -1 & 1 & 1 & -1 & -1 & -1 & 1 & 1 \\
\hline & & & & & & & 3 & -1 & -1 & 1 & 1 & 1 & -1 & -1 & 1 \\
\hline \multirow{3}{*}{6} & \multirow{3}{*}{4} & \multirow{3}{*}{+1} & \multirow{3}{*}{-1} & \multirow{3}{*}{+1} & \multirow{3}{*}{-1} & & 1 & 1 & 1 & -1 & -1 & 1 & 1 & -1 & -1 \\
\hline & & & & & & & 2 & 1 & 1 & -1 & -1 & -1 & 1 & 1 & -1 \\
\hline & & & & & & & 3 & 1 & -1 & -1 & 1 & 1 & 1 & -1 & -1 \\
\hline 7 & 5 & -1 & +1 & -1 & +1 & -1 & 1 & -1 & 1 & 1 & -1 & -1 & 1 & 1 & -1 \\
\hline 8 & 5 & +1 & -1 & +1 & -1 & +1 & 1 & 1 & -1 & -1 & 1 & 1 & -1 & -1 & 1 \\
\hline
\end{tabular}
уровней факторов $(\mu)$. Подробно процедура построения описана в работе [4].

4. Базовые серийные последовательности для $n=8, k=3, \mu=7$

5. Типовые варианты структур оптимальных планов МФЭ для $n=8, k=3, \mu=7$

\begin{tabular}{|c|c|c|c|c|c|c|c|c|c|c|c|}
\hline № & $\mathrm{A}_{1}$ & $\mathrm{~A}_{2}$ & $\mathrm{~A}_{3}$ & № & $\mathrm{A}_{1}$ & $\mathrm{~A}_{2}$ & $\mathrm{~A}_{3}$ & № & $\mathrm{A}_{1}$ & $\mathrm{~A}_{2}$ & $\mathrm{~A}_{3}$ \\
\hline 1 & $4(1)$ & $6(1)$ & $4(2)$ & 2 & $1(1)$ & $8(1)$ & $4(3)$ & 3 & $4(2)$ & $5(1)$ & $3(1)$ \\
\hline 4 & $1(1)$ & $4(3)$ & $7(1)$ & 5 & $1(1)$ & $5(2)$ & $6(2)$ & 6 & $1(1)$ & $5(2)$ & $5(3)$ \\
\hline 7 & $1(1)$ & $8(1)$ & $3(2)$ & 8 & $4(1)$ & $5(1)$ & $3(3)$ & 9 & $1(1)$ & $5(3)$ & $6(3)$ \\
\hline 10 & $5(2)$ & $5(3)$ & $2(1)$ & 11 & $4(1)$ & $4(2)$ & $5(1)$ & 12 & $8(1)$ & $4(3)$ & $2(1)$ \\
\hline 13 & $1(1)$ & $7(1)$ & $3(2)$ & 14 & $1(1)$ & $6(2)$ & $6(3)$ & 15 & $6(1)$ & $3(1)$ & $3(3)$ \\
\hline 16 & $5(1)$ & $3(1)$ & $3(3)$ & 17 & $7(1)$ & $3(2)$ & $2(1)$ & 18 & $6(2)$ & $6(3)$ & $2(1)$ \\
\hline 19 & $4(1)$ & $6(1)$ & $3(3)$ & 20 & $4(3)$ & $7(1)$ & $2(1)$ & 21 & $5(2)$ & $6(2)$ & $2(1)$ \\
\hline 22 & $6(1)$ & $4(2)$ & $3(1)$ & 23 & $8(1)$ & $3(2)$ & $2(1)$ & 24 & $5(3)$ & $6(3)$ & $2(1)$ \\
\hline
\end{tabular}


Пример каталога типовых оптимальных планов МФЭ для $\mathrm{n}=8, \mathrm{k}=3, \mu=7$ приведен ниже. Базовые серийные последовательности приведены в таблице 4. Типовые варианты структур оптимальных планов МФЭ приведены в таблице 5 . При описании типа указывается номер базовой серийной последовательности, которая используется, и номер вида последовательности.

Предложенный метод перечисления позволяет строить каталоги оптимальных планов МФЭ, которые используются для выбора плана МФЭ с минимальной стоимостью изменений уровней факторов.

Вывод. Предложен метод перечисления типовых оптимальных планов МФЭ, основанный на

\section{БИБЛИОГРАФИЯ}

1. Комевой Н. Д. Применение комбинаторного анализа при выборе оптимальных планов многофакторного эксперимента / Н. Д. Кошевой, С. Г. Бестань, В. А. Дергачев // Теорія і практика перебудови економіки : зб. наукових праць ЧІТІ. Черкаси, 2001. - С. 224-227.

2. Кошевой Н. Д. Эквивалентные преобразования комбинаторных планов многофакторного эксперимента / Н. Д. Кошевой, В. П. Сироклын, А. В. Дергачева // Вісник Черкаського державного технологічного університету : Спецвипуск. Черкаси : ЧДТУ, 2006. - С. 163-165.

3. Комевой Н. Д. Классификация планов многофакторного эксперимента / Н. Д. Кошевой, А. В. Павлик, В. П. Сироклын // Збірник наукових праць Харківського університету повітряних сил. - Х., 2008. - Вып. 1 (16). - С. 65-67.

4. Кочевой Н. Д. Метод классификации планов многофакторного эксперимента / М.В.Цехов- представлении планов МФЭ в виде серийных последовательностей. Рассмотрено решение поэтапных задач метода. Исследованы свойства серийных последовательностей планов МФЭ, получены оценки их количества, описана процедура формирования множества базовых серийных последовательностей и типовых вариантов структур планов МФЭ. Приведены примеры каталогов оптимальных планов МФЭ. Применение предложенного метода позволит упростить процесс выбора минимальных планов МФЭ для большого количества факторов. Следующим этапом исследований является разработка алгоритмического и программного обеспечения для автоматизации решения рассмотренной задачи.

ской, Н. Д. Кошевой, В. А. Дергачев, Е. М. Костенко // Радіоелектронні і комп'ютерні системи. - 2011. - №3(51). - С. 108-112.

5. Курмаев О. Ф. Кодирование последовательностей с ограниченными длинами серий // Пробл. передачи информ. - 2001. - Т. 37; № 3. C. 34-43.

6. Маркова E. В. Комбинаторные планы в задачах многофакторного эксперимента / Е. В. Маркова, А. Н. Лисенков. - М. : Наука, 1979. - 347 с. 7. Таблицы планов эксперимента для факторных и полиномиальных моделей : справочн. изд. / В. З. Бродский, Л. И. Бродский, Т. И. Голикова [и др.] / Ред. Налимова В. В. - М. : Металлургия, 1982. $-750 \mathrm{c}$.

8. Roytberg M. A. A Search for Common Patterns in many Sequences // Comput. Appl. Biosci. - 1992. Vol. 8, № 1. - P. 57-64. 